American J. of Engineering and Applied Sciences 4 (1): 37-41, 2011

ISSN 1941-7020

(C) 2010 Science Publications

\title{
Weighted Centroid Correction Localization in Cellular Systems
}

\author{
Rong-Zheng Li, Xin-Long Luo and Jia-Ru Lin \\ Key Laboratory of Universal Wireless Network, \\ School of Information and Communication Engineering, \\ Beijing University of Posts and Telecommunications, China
}

\begin{abstract}
Problem statement: There is a large demand for wireless Location-Based Service (LBS) and it is provided by many wireless cellular systems. In process of positioning a Mobile Station (MS), the computing speed is as important as the positioning accuracy and the algorithm should also be resistant to environmental influences. Approach: A new positioning method based on Weighted Centroid Correction Localization (WCCL) for wireless cellular systems is introduced in this article. Firstly, referring to the receiving-state of an MS in cellular systems, it computes a weighted centroid of surrounding Base Stations (BSs) as a rough approximate position of the MS. Then, according to the distances between the MS and the BSs being less or bigger than the computed distances between the BSs and the weighted centroid, it corrects the coordinate of the weighted centroid towards the directions of the BSs by moving it closer or farther in turn. Results: According to our experiments, WCCL improves the positioning accuracy, as well as to provide a better resistance to environmental influences. Conclusion: As a modified centroid-based localization algorithm, WCCL obtains weighting factors from the receiving-state of MS in multi-cells structured cellular systems and obtains a better positioning result in cellular systems without updating the network equipment. Therefore, for the cellular positioning problem, WCCL algorithm can be an alternate solution.
\end{abstract}

Key words: Wireless positioning method, Location-Based Service (LBS), geometric distance, Base Stations (BSs), weighted centroid correction localization (WCCL), cellular systems, Arrival Of Angle (AOA), Location Measurement Unit (LMU), Effective Cells (ECs)

\section{INTRODUCTION}

There is a large demand for wireless Location-Based Service (LBS) and it is provided by many wireless cellular systems (Reed et al., 1998; Drane et al., 1998; Basi and Murthy, 2004). In process of positioning a Mobile Station (MS), the computing speed is as important as the positioning accuracy and the algorithm should also be resistant to environmental influences.

Cell-ID method is the first cellular positioning technology applied to commercial services. It adopts the coordinate of the Base Station (BS) which has the strongest signal strength as the position of the mobile station, but it only has a low accuracy (Caffery and Stuber, 1998; Drane et al., 1998). Another positioning method is the triangle algorithm, which obtains the positioning coordinate of an MS by solving a linear least-square problem based on the distances between the MS and surrounding BSs (Luo and $\mathrm{Wu}, 2010$; Zheng et al., 2008). However, the positioning accuracy of the triangle method is very sensitive to the precision of measured distances.

In order to obtain a better positioning result, other positioning technologies are developed in recent years, such as assisted GPS, Arrival Of Angle (AOA), Time Of Arrival/Time Difference Of Arrival (TOA/TDOA) (Caffery and Stuber, 1998; Drane et al., 1998). The positioning technology based on the assisted GPS or AOA needs to deploy the special hardware (GPS chip or antenna array) in an MS or a BS. If a non-synchronous system tries to locate an MS via TOA/TDOA, it needs to deploy Location Measurement Unit (LMU) to improve the timing capability and the MS also must be upgraded. Another disadvantage, they usually take much more time to respond the request of the positioning because of their complex computing process.

Therefore, we need to investigate a simple and efficient method to improve the positioning accuracy with less time cost, as well as to provide a better resistance to environmental influences. The weighted

Corresponding Author: Xin-long Luo, Key Laboratory of Universal Wireless Network, School of Information and Communication Engineering, Beijing University of Posts and Telecommunications, Beijing 100876, China 
centroid-based localization algorithm is a good backup approach because of its simplicity and efficiency, on the recent advancement of this class of positioning methods, one can refer to (Blumenthal et al., 2007) for the Weighted Centroid Localization (WCL), (Behnke and Timmermann, 2008) for the adaptive WCL (AWCL), (Schuhmann et al., 2010) for the improved WCL (IWCL) and so on.

\section{MATERIALS AND METHODS}

In this study, we proposed a new localization algorithm for wireless cellular systems, which is called the Weighted Centroid Correction Localization (WCCL). Compared with other weighted centroidbased algorithms, the different characteristic of WCCL is that its weighting factors are obtained from the receiving-state of an MS in multi-cells structured cellular systems. In order to improve the positioning accuracy of an MS, we use the distances between the MS and surrounding BSs to correct its primary computed position.

In wireless cellular systems, directional antennas are widely deployed and every BS usually has three or four directional antennas and each antenna aims toward different direction (Takai et al., 2002; Vasquez and Hao, 2001). We usually use a cell to express the electromagnetic wave transmitted by an antenna. If an MS is in the coverage of a BS, it may receive signals from multi-cells of that BS. We call those cells as Effective Cells (ECs) and call that BS as an Effective Base Station (EBS).

If an isolated BS has three directional antennas which aim to three different directions with the spacing of $120^{\circ}$ respectively, the coverage of three antennas overlap each other in some regions. We divided the coverage of a BS into several regions which are marked by A-G, as depicted in Fig. 1.

The coverage radius of main lobe $\mathrm{R}_{3}$ is bigger than back-lobe's coverage radius $R_{1}$ since the gain of an antenna's main-lobe is stronger than that of back-lobe. $\mathrm{R}_{2}$ is a boundary of the two types of regions, where an MS can receive signals from two antennas or one antenna. Therefore, the receiving-states of an MS in different regions A-G are listed in Table 1.

From Table 1, we can conclude that the number of effective cells is 3 when an MS is in a region of $R_{1}$ and the number of effective cells is 2 if the distance between the MS and the BS ranges from $R_{1}-R_{2}$ and the number of effective cells is 1 if the MS is beyond the region of $\mathrm{R}_{2}$ but still in the coverage of the BS.

In a cellular system, in order to guarantee the continuous coverage of a network and the successful

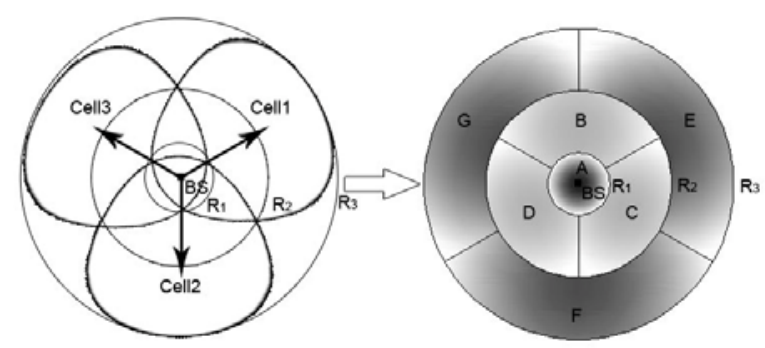

Fig. 1: The analysis of a base station's coverage

handover of an MS, the spacing between BSs is usually set within the range from $R_{2}-R_{3}$. Therefore, we take the number of effective cells of a BS as a weighting factor of that BS. This basic feature is important for WCCL.

Publicized algorithms of the centroid location methods use the centroid to calculate the position of an MS (Blumenthal et al., 2007; Schuhmann et al., 2010; Bulusu et al., 2000). For example, an MS receives signals from multi-cells at the position $\mathrm{M}(\mathrm{x}, \mathrm{y})$ and the cells belong to BSs with positions of $\mathrm{B}_{\mathrm{i}}(\mathrm{x}, \mathrm{y})$ $\left(i=1,2, \ldots, n\right.$.), then the approximated position $M^{\prime}(x, y)$ of the MS can be calculated by Eq. 1:

$M^{\prime}(x, y)=\frac{1}{n} \sum_{i=1}^{n} B_{i}(x, y)$

The positioning error e is defined as the distance between the exact position $\mathrm{M}(\mathrm{x}, \mathrm{y})$ and the approximated position $\mathrm{M}^{\prime}$ ( $\left.\mathrm{x}, \mathrm{y}\right)$ of an MS by Eq. 2:

$e=\sqrt{\left(x^{\prime}-x\right)^{2}+\left(y^{\prime}-y\right)^{2}}$

A centroid location method only uses the average of the coordinates of BSs to approximate the location of an MS. A WCL (Blumenthal et al., 2007) uses the weighted coefficients to ensure an improved localization which is formulated by Eq. 3:

$\mathrm{M}^{\prime \prime}(\mathrm{x}, \mathrm{y})=\frac{\sum_{\mathrm{i}=1}^{\mathrm{n}}\left\{\mathrm{w}_{\mathrm{i}} \bullet \mathrm{B}_{\mathrm{i}}(\mathrm{x}, \mathrm{y})\right\}}{\sum_{\mathrm{i}=1}^{\mathrm{n}} \mathrm{w}_{\mathrm{i}}}$

where the weight $\mathrm{w}_{\mathrm{i}}$ is defined by:

$\mathrm{w}_{\mathrm{i}}=\frac{1}{\left(\mathrm{~d}_{\mathrm{i}}\right)^{\mathrm{g}}} \quad\left(\mathrm{d}_{\mathrm{i}}=\right.$ distance between MS and $\mathrm{BS}_{\mathrm{i}}, \mathrm{g}=$ degree $)$ 
Am. J. Engg. \& Applied Sci., 4 (1): 37-41, 2011

Table 1: Receiving-states of a MS in regions A-G

\begin{tabular}{llllll}
\hline Region & Cell $_{1}$ & Cell $_{2}$ & Cell $_{3}$ & Num of ECs & $d$ \\
\hline A & received & received & received & 3 & $d<R_{1}$ \\
B & received & - & received & 2 & $R_{1}<d<R_{2}$ \\
C & received & received & - & 2 & $R_{1}<d<R_{2}$ \\
D & - & received & received & 2 & $R_{1}<d<R_{2}$ \\
E & received & - & - & 1 & $R_{2}<d<R_{3}$ \\
F & - & received & - & 1 & $R_{2}<d<R_{3}$ \\
G & - & - & received & 1 & $R_{2}<d<R_{3}$ \\
\hline
\end{tabular}

In order to improve the positioning accuracy, we give a new centroid-based localization algorithm, i.e., the Weighted Centroid Correction Localization (WCCL). Its weighted factor $\mathrm{w}_{\mathrm{i}}$ is obtained from the receiving-state of an MS in multi-cells structured cellular systems. The approximated weighted centroid $\mathrm{M}_{\mathrm{wc}}(\mathrm{x}, \mathrm{y})$ is computed by Eq. 5 , where the weight of the i-th BS $w_{i}$ equals the number of effective cells which belong to that $\mathrm{BS}$ :

$\mathrm{M}_{\mathrm{wc}}(\mathrm{x}, \mathrm{y})=\frac{\sum_{\mathrm{i}=1}^{\mathrm{n}}\left\{\mathrm{w}_{\mathrm{i}}^{\prime} \bullet \mathrm{B}_{\mathrm{i}}(\mathrm{x}, \mathrm{y})\right\}}{\sum_{\mathrm{i}=1}^{\mathrm{n}} \mathrm{w}_{\mathrm{i}}^{\prime}}$

Then, according to the distance $d_{i}$ between the $i$-th $\mathrm{BS}_{\mathrm{i}}(\mathrm{x}, \mathrm{y})$ and the MS M(x,y) being less or bigger than the computed distance $\mathrm{d}^{\prime}{ }_{\mathrm{i}}$ between that $\mathrm{BS} \mathrm{B}_{\mathrm{i}}(\mathrm{x}, \mathrm{y})$ and the weighted centroid $\mathrm{M}_{\mathrm{wc}}(\mathrm{x}, \mathrm{y})$, we correct the coordinate of the weighted centroid towards the direction of the i-th BS by moving it closer or farther. The above process is executed for every the effective base station by turns. Therefore, the final corrected position $\mathrm{M}_{\mathrm{wcc}}(\mathrm{x}, \mathrm{y})$ is obtained by:

$$
\mathrm{M}_{\mathrm{wcc}}(\mathrm{x}, \mathrm{y})=\mathrm{M}_{\mathrm{wc}}(\mathrm{x}, \mathrm{y})+\sum_{\mathrm{i}=1}^{\mathrm{n}}\left\{\mathrm{v}_{\mathrm{n}} \bullet\left(\mathrm{d}_{\mathrm{i}}^{\prime}-\mathrm{d}_{\mathrm{i}}\right) \bullet \frac{\overline{\mathrm{M}_{\mathrm{wc}} \mathrm{B}_{\mathrm{i}}}}{\left.\mid \overline{\mathrm{M}_{\mathrm{wc}} \mathrm{B}_{\mathrm{i}} \mid}\right\}}\right\}
$$

where, $v_{n}$ is a correcting factor which relates with the number of effective base stations.

\section{RESULTS}

In order to verify the performance of positioning methods in real cellular network, we choose a region with $4 \times 2 \mathrm{~km}$ around Jinmen Bridge in Haidian District Beijing China as a test environment. The test mobile station moves along seven main roads which are indicated in Fig. 2. Those test experiments were performed in a GSM network and we obtained 3744 valid test points.
Table 2: Statistical results of test points

\begin{tabular}{llll}
\hline Num of EBSs & Color & Quantity & Percentage \\
\hline 1 & red & 119 & $3.18 \%$ \\
2 & yellow & 1033 & $27.59 \%$ \\
3 & green & 1500 & $40.06 \%$ \\
$4-7$ & blue & 1092 & $29.17 \%$ \\
\hline
\end{tabular}

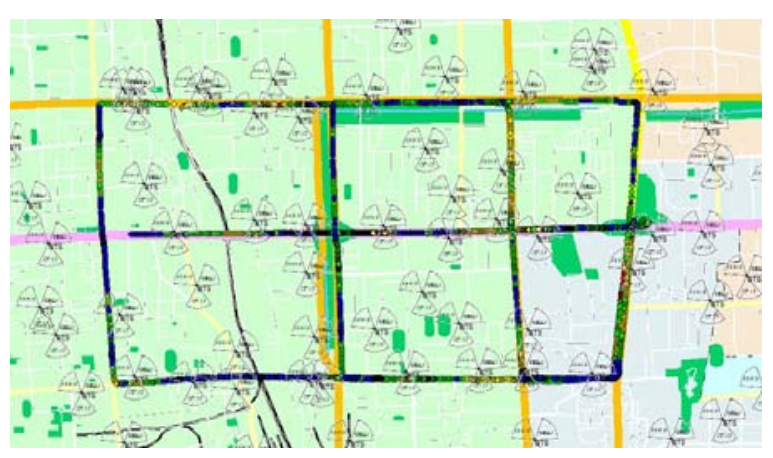

Fig. 2: Distribution of test points and base stations

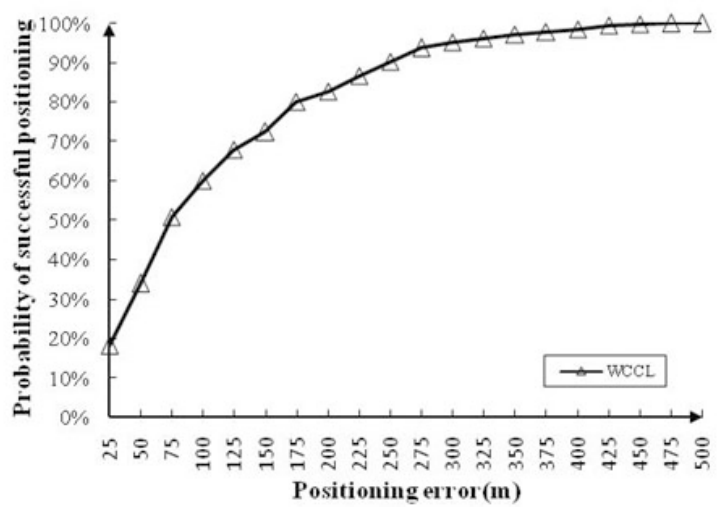

Fig. 3: CDF of positioning error by WCCL in experimental model

The test mobile station can receive wireless signals from seven cells at most, some of which may belong to same BSs. We divided those test points into four classes according to the Num of EBSs and the different classes of test points are marked by different colors in Fig. 2, respectively. Their statistical results are listed in Table 2. 
Am. J. Engg. \& Applied Sci., 4 (1): 37-41, 2011

Positioning error is the geometric distance between the exact position $\mathrm{M}(\mathrm{x}, \mathrm{y})$ and the approximated position $\mathrm{M}_{\mathrm{wcc}}(\mathrm{x}, \mathrm{y})$ of an MS computed by (5-6). We chose the parameters $\mathrm{v}_{2}=0.5, \mathrm{v}_{3}=0.5$ and $\mathrm{v}_{\mathrm{i}}=0.4(\mathrm{i}=4,5,6,7)$ in (6). The Cumulative Distribution Function (CDF) of the positioning error by WCCL in experimental model is shown by Fig. 3 and the positioning error achieves 120 meters with the successful positioning probability of $67 \%$.

\section{DISCUSSION}

The distance $d_{i}$ between an MS and the i-th BS is very important for triangle algorithm, WCL and WCCL. Because of multipath and non-light-of-sight parameters, the estimating error is inevitably. We denote the exact distance between an MS and the i-th base station as $\mathrm{d}_{0 \mathrm{i}}$ and the estimating error $\Delta \mathrm{d}_{\mathrm{i}}$ reflects the deviation level from $d_{0 \mathrm{i}}$ to $\mathrm{d}_{\mathrm{i}}$, which is obtained by:

$$
\Delta \mathrm{d}_{\mathrm{i}}=\left|\mathrm{d}_{0 \mathrm{i}}-\mathrm{d}_{\mathrm{i}}\right|
$$

The precision of the estimated distance affects the accuracy of positioning. Therefore a good positioning algorithm should be resistant to environmental influences as well as imprecise distances. In order to quantify environmental impacts, we propose a parameter $R_{\Delta d \max }$ defined by (8), which is called the maximum relative error rate of estimated distance:

$$
\Delta \mathrm{d}_{\mathrm{i}} \in\left[0, \mathrm{~d}_{0 \mathrm{i}} \bullet \mathrm{R}_{\Delta \mathrm{d} \max }\right]
$$

In order to compare WCCL with other popular positioning algorithms, we estimate the coordinates of all the test points with more than two EBSs, by cell-ID, CL, WCL, WCCL and the triangle algorithm, respectively. Figure 4 illustrates the positioning errors with the probability of $67 \%$ by different localization algorithm in our experimental model and the curves show the trends of positioning errors by different localization algorithms, which change with the increasing of $\mathrm{R}_{\Delta \mathrm{dmax}}$.

From Fig. 4, owing to the absence of distance information in localization computing, we find that cellID and CL can only roughly localize the coordinate of an MS with low precision. The triangle algorithm can obtain a good positioning result if the distance estimating error is low and the positioning error will deteriorate sharply with the increasing of $R_{\Delta d \max }$. Therefore, the triangle algorithm cannot be resistant to environmental influences.

WCL algorithm improves the positioning accuracy of an MS and the positioning error can be reduced to

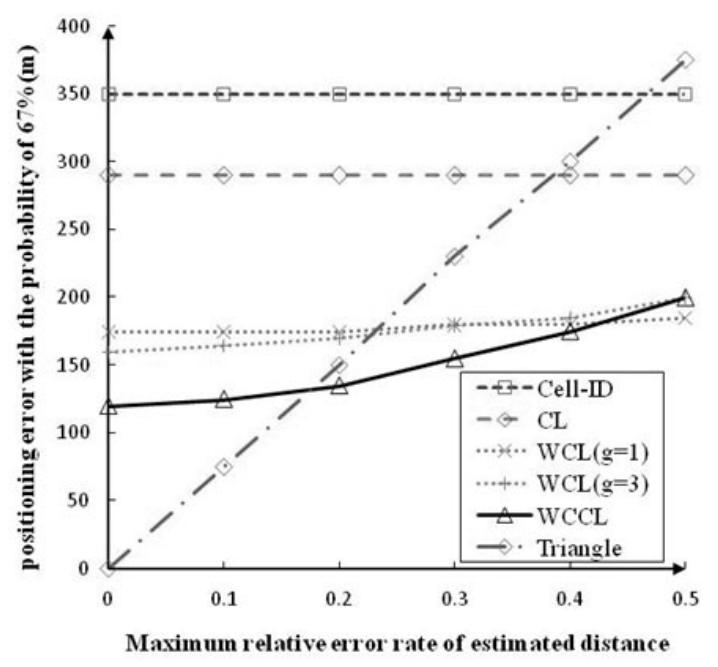

Fig. 4: Positioning errors with the probability of $67 \%$ by different localization algorithms in experimental model

almost a half from CL with the probability of $67 \%$ and also be resistant to the increasing of $R_{\Delta d \max }$.

As a new centroid-based localization algorithm, WCCL obtains weighting factor $w_{i}{ }_{i}$ from the receivingstate of an MS in multi-cells structured cellular systems which is discussed in the second section and uses the distances between the MS and BSs to correct the approximated weighted centroid. As is illustrated in Fig. 4, WCCL algorithm can reduce the positioning error by near $30 \%$ from WCL with the probability of $67 \%$ and Fig. 4 also shows that the position accuracy of WCCL is not sensitive to the maximum relative error $\mathrm{R}_{\Delta \mathrm{dmax}}$. Before $\mathrm{R}_{\Delta \mathrm{dmax}}$ increases to 0.5 , the positioning result of WCCL is always much better than that of WCL.

\section{CONCLUSION}

In comparison with several other popular positioning methods, WCCL obtains a better positioning result in cellular systems and it also shows a good resistance to environmental influences. Furthermore, WCCL can be easily implemented in a wireless cellular system and it does not need to update the equipment of the network or MS. Therefore, for the cellular positioning problem, WCCL algorithm can be an alternate solution.

\section{ACKNOWLEDGEMENT}

This study is supported by Grant YBWL2010152 from Research Fund for Huawei Technologies Co., Ltd 
and Grant BUPT2009RC0118 from the Fundamental Research Funds for the Central Universities.

\section{REFERENCES}

Basi, H.M.A. and M.B.R. Murthy, 2004. A simple scheme for improved performance of fixed outage rate cellular system. Am. J. Applied Sci., 1: 190-192. DOI: $10.3844 / .2004 .190 .192$

Behnke, R. and D. Timmermann, 2008. AWCL: Adaptive weighted centroid localization as an efficient improvement of coarse grained localization. Proceeding of the WPNC 5th Workshop on Positioning, Navigation and Communication, March 27-27, IEEE Xplore Press, Hannover, pp: 243-250.

Blumenthal, J., R. Grossmann, F. Golatowski and D. Timmermann, 2007. Weighted centroid localization in zigbee-based sensor networks. Proceeding of the IEEE International Symposium on Intelligent Signal Processing, Oct. 3-5, IEEE Xplore Press, Alcala de Henares, pp: 1-6. DOI: 10.1109/WISP.2007.4447528

Bulusu, N., J. Heidemann and D. Estrin, 2000. GPSless low-cost outdoor localization for very small devices. IEEE Personal Commun. Maga., 7: 28-34. DOI: $10.1109 / 98.878533$

Caffery, J.J. and G.L. Stuber, 1998. Overview of radiolocation in CDMA cellular systems. IEEE Commun. Maga., 36: 38-45. DOI: 10.1109/35.667411

Drane, C., M. Macnaughtan and C. Scott, 1998. Positioning GSM telephones, IEEE Commun. Maga., 36: 46-54. DOI: 10.1109/35.667413
Luo, X.L. and Z.J. Wu, 2010. Least-squares approximations in geometric buildup for solving distance geometry problems, J. Optim. Theory Appl., Accepted. http://orion.math.iastate.edu/wu/least09luo.pdf

Reed, J.H., K.J. Krizman, B.D. Woerner and T.S. Rappaport, 1998. An overview of the challenges and progress in meeting the E-911 requirement foe location service. IEEE Commun. Maga., 36: 30-37. DOI: 10.1109/35.667410

Schuhmann, S., K. Herrmann, K. Rothermel, J. Blumenthal and D. Timmermann, 2010. Improved weighted centroid localization in smart ubiquitous environments. Ubiquitous Intell. Comput., 5610: 20-34. DOI: 10.1007/978-3-540-69293-5 4

Takai, M., J. Martin, R. Bagrodia and A. Ren, 2002. Directional virtual carrier sensing for directional antennas in mobile ad hoc networks. Proceedings of the 3rd ACM International Symposium on Mobile Ad Hoc Networking and Computing, (ISMAHNC' 02), ACM MobiHoc, USA., pp: 183-193. DOI: $10.1145 / 513800.513823$

Vasquez, M. and J.K. Hao, 2001. A heuristic approach for antenna positioning in cellular networks. J. Heuristics, 7: 443-472. DOI: 10.1023/A:1011373828276

Zheng, Z., X. Luo and Z. Wu, 2008. A Two-phase geometric buildup algorithm for the solution of the sensor network localizationm. ACM Transactions on Computational Logic, 20: 111-127. http://orion.math.iastate.edu/wu/sensor09.pdf 